\title{
Finite Domain Bounds Consistency Revisited
}

\author{
C.W. Choi* $\quad$ W. Harvey ${ }^{\dagger} \quad$ J.H.M. Lee* $\quad$ P. J. Stuckey ${ }^{\ddagger}$
}

August 3, 2018

\begin{abstract}
A widely adopted approach to solving constraint satisfaction problems combines systematic tree search with constraint propagation for pruning the search space. Constraint propagation is performed by propagators implementing a certain notion of consistency. Bounds consistency is the method of choice for building propagators for arithmetic constraints and several global constraints in the finite integer domain. However, there has been some confusion in the definition of bounds consistency. In this paper we clarify the differences and similarities among the three commonly used notions of bounds consistency.
\end{abstract}

\section{Introduction}

Finite domain constraint programming combines backtracking tree search with constraint propagation to solve constraint satisfaction problems (CSPs) [14. This framework is realized in constraint programming systems, such as ECL ${ }^{i} \mathrm{PS}^{e}$, 4], SICStus Prolog 22] and ILOG Solver [11, which have been successfully applied to many real-life industrial applications.

Constraint propagation, based on local consistency algorithms, removes infeasible values from the domains of variables to reduce the search space. The origins of this technique in the Artificial Intelligence field concentrated on consistency notions for constraints with two or fewer variables. The most successful consistency technique was arc consistency [14] which ensures that for each constraint, every value in the domain of one variable has a supporting value in the domain of the other variable which satisfies the constraint.

Arc consistency can be naturally extended to constraints of more than two variables. This extension has been called generalized arc consistency [17, as well as domain consistency [23] (which is the terminology we will use), and

\footnotetext{
${ }^{*}$ Department of Computer Science and Engineering, The Chinese University of Hong Kong, Shatin, N.T., Hong Kong SAR, China. Email: \{cwchoi,jlee\}@cse.cuhk.edu.hk

${ }^{\dagger}$ IC-Parc, William Penney Laboratory, Imperial College London, Exhibition Road, London SW7 2AZ. Email: wh@icparc.ic.ac.uk

${ }^{\ddagger}$ NICTA Victoria Laboratory, Department of Computer Science \& Software Engineering, University of Melbourne, 3010, Australia. Email: pjs@cs.mu.oz.au
} 
hyper-arc consistency [16]. Checking domain consistency is NP-complete even for linear equations, an important kind of constraint.

To avoid this problem, weaker forms of consistency were introduced for handling constraints with large numbers of variables. The most notable one for linear arithmetic constraints has been bounds consistency (sometimes called interval consistency). Unfortunately there are three commonly used but incompatible definitions of bounds consistency in the literature. This is confusing to practitioners in the design and implementation of efficient bounds consistency algorithms, as well as for users of constraint programming system claiming to support bounds consistency. In this paper we clarify the three existing definitions of bounds consistency and the differences between them. We also gives conditions under which the definitions may coincide for certain constraints.

\section{Background}

In this paper we consider integer constraint solving using constraint propagation. Let $\mathcal{Z}$ denote the integers, and $\mathcal{R}$ denote the reals. Hence $\mathcal{Z} \models F$ denotes that the formula $F$ is universally true in the integers (that is when the constants, functions and variables are interpreted in the integer domain). Similarly $\mathcal{R} \models F$ denotes that the formula $F$ is universally true in the reals.

We consider a given (finite) set of integer variables $\mathcal{V}$, which we shall sometimes interpret as real variables. Each variable is associated with a finite set of possible values, defined by the domain. A domain $D$ is a complete mapping from a set of variables $\mathcal{V}$ to finite sets of integers. We assume in this paper that $D(v)$ for all $v \in \mathcal{V}$ are totally ordered.

A valuation $\theta$ is a mapping of variables to values (integers or reals), written $\left\{x_{1} \mapsto d_{1}, \ldots, x_{n} \mapsto d_{n}\right\}$. Let vars be the function that returns the set of variables appearing in an expression, constraint or valuation. Given an expression $e, \theta(e)$ is obtained by replacing each $v \in \operatorname{vars}(e)$ by $\theta(v)$ and calculating the value of the resulting variable free expression.

In an abuse of notation, we define a valuation $\theta$ to be an element of a domain $D$, written $\theta \in D$, iff $\theta(v) \in \mathcal{Z}$ and $\theta(v) \in D(v)$ for all $v \in \operatorname{vars}(\theta)$. We are interested in determining the infimums and supremums of expressions with respect to some domain $D$. Define the infimum and supremum of an expression $e$ with respect to a domain $D$ as $\inf _{D} e=\inf \{\theta(e) \mid \theta \in D\}$ and $\sup _{D} e=\sup \{\theta(e) \mid \theta \in D\}$. We also use range notation: $[l . . u]$ denotes the set $\{d \in \mathcal{Z} \mid l \leq d \leq u\}$ when $l$ and $u$ are integers. A domain is a range domain if $D(x)$ is a range for all $x$. Let $D^{\prime}=\operatorname{range}(D)$ be the smallest range domain containing $D$, i.e. domain $D^{\prime}(x)=\left[\inf _{D} x . . \sup _{D} x\right]$ for all $x \in \mathcal{V}$.

A constraint places restriction on the allowable values for a set of variables and is usually written in well understood mathematical syntax. More formally, a constraint $c$ is a relation expressed using available function and relation symbols in a specific constraint language. For the purpose of this paper, we assume the usual integer interpretation of arithmetic constraints and logical operators such as $\neg, \wedge, \vee, \Rightarrow$, and $\Leftrightarrow$. We call $\theta$ an integer solutions of $c$ iff $\operatorname{vars}(\theta)=\operatorname{vars}(c)$ 
and $\mathcal{Z} \models_{\theta} c$. Similarly, we call $\theta$ a real solutions of $c$ iff $\operatorname{vars}(\theta)=\operatorname{vars}(c)$ and $\mathcal{R} \models_{\theta} c$. A constraint satisfaction problem (CSP) consists of a set of constraints read as conjunction.

Constraint propagation enforcing domain consistency ensures that for each constraint $c$, every value in the domain of each variable can be extended to an assignment satisfying $c$.

Definition 1 A domain $D$ is domain consistent for a constraint $c$ where vars $(c)=$ $\left\{x_{1}, \ldots, x_{n}\right\}$, if for each variable $x_{i}, 1 \leq i \leq n$ and for each $d_{i} \in D\left(x_{i}\right)$ there exist integers $d_{j}$ with $d_{j} \in D\left(x_{j}\right), 1 \leq j \leq n, j \neq i$ such that $\theta=\left\{x_{1} \mapsto\right.$ $\left.d_{1}, \ldots, x_{n} \mapsto d_{n}\right\}$ is an integer solution of $c$.

Example 2 Consider the constraint $c_{\text {lin }} \equiv x_{1}=3 x_{2}+5 x_{3}$. The domain $D_{0}$ defined by $D_{0}\left(x_{1}\right)=[2 . .7], D_{0}\left(x_{2}\right)=[0 . .2]$, and $D_{0}\left(x_{3}\right)=[-1 . .2]$ is not domain consistent w.r.t. $c_{l i n}$. But the domain $D_{1}$ defined by $D_{1}\left(x_{1}\right)=\{3,5,6\}$, $D_{1}\left(x_{2}\right)=\{0,1,2\}$, and $D_{1}\left(x_{3}\right)=\{0,1\}$ is domain consistent for $c_{l i n}$, supported by the integer solutions $\theta_{1}=\left\{x_{1} \mapsto 3, x_{2} \mapsto 1, x_{3} \mapsto 0\right\}, \theta_{2}=\left\{x_{1} \mapsto 5, x_{2} \mapsto\right.$ $\left.0, x_{3} \mapsto 1\right\}$, and $\theta_{3}=\left\{x_{1} \mapsto 6, x_{2} \mapsto 2, x_{3} \mapsto 0\right\}$.

\section{Different Notions of Bounds Consistency}

The basis of bounds consistency is to relax the consistency requirement to apply only to the lower and upper bounds of the domain of each variable. There are three incompatible definitions of bounds consistency used in the literature, all for constraints with finite integer domains. The first and second definitions are based on integer solutions, while the third definition is based on real solutions.

Definition $3 A$ domain $D$ is bounds $(\mathcal{D})$ consistent for a constraint $c$ where $\operatorname{vars}(c)=\left\{x_{1}, \ldots, x_{n}\right\}$, if for each variable $x_{i}, 1 \leq i \leq n$ and for each $d_{i} \in$ $\left\{\inf _{D} x_{i}, \sup _{D} x_{i}\right\}$ there exist integers $\boldsymbol{d}_{\boldsymbol{j}}$ with $\boldsymbol{d}_{\boldsymbol{j}} \in \boldsymbol{D}\left(\boldsymbol{x}_{\boldsymbol{j}}\right), 1 \leq j \leq n, j \neq i$ such that $\theta=\left\{x_{1} \mapsto d_{1}, \ldots, x_{n} \mapsto d_{n}\right\}$ is an integer solution of $c$.

Definition $4 A$ domain $D$ is bounds $(\mathcal{Z})$ consistent for a constraint $c$ where $\operatorname{vars}(c)=\left\{x_{1}, \ldots, x_{n}\right\}$, if for each variable $x_{i}, 1 \leq i \leq n$ and for each $d_{i} \in$ $\left\{\inf _{D} x_{i}, \sup _{D} x_{i}\right\}$ there exist integers $\boldsymbol{d}_{\boldsymbol{j}}$ with $\inf _{\boldsymbol{D}} \boldsymbol{x}_{\boldsymbol{j}} \leq \boldsymbol{d}_{\boldsymbol{j}} \leq \sup _{\boldsymbol{D}} \boldsymbol{x}_{\boldsymbol{j}}, 1 \leq$ $j \leq n, j \neq i$ such that $\theta=\left\{x_{1} \mapsto d_{1}, \ldots, x_{n} \mapsto d_{n}\right\}$ is an integer solution of $c$.

Definition $5 A$ domain $D$ is bounds $(\mathcal{R})$ consistent for a constraint $c$ where $\operatorname{vars}(c)=\left\{x_{1}, \ldots, x_{n}\right\}$, if for each variable $x_{i}, 1 \leq i \leq n$ and for each $d_{i} \in$ $\left\{\inf _{D} x_{i}, \sup _{D} x_{i}\right\}$ there exist real numbers $\boldsymbol{d}_{\boldsymbol{j}}$ with $\inf _{\boldsymbol{D}} \boldsymbol{x}_{\boldsymbol{j}} \leq \boldsymbol{d}_{\boldsymbol{j}} \leq \sup _{\boldsymbol{D}} \boldsymbol{x}_{\boldsymbol{j}}$, $1 \leq j \leq n, j \neq i$ such that $\theta=\left\{x_{1} \mapsto d_{1}, \ldots, x_{n} \mapsto d_{n}\right\}$ is a real solution of $c$.

Definition [3] is used in for example for the two definitions in Dechter 7 , pages 73 \& 435]; Frisch et al. [8]; and implicitly in Lallouet et al. [12. Definition 4 is far more widely used appearing in for example Van Hentenryck et 
al. 23]; Puget [18; Régin \& Rueher [20]; Quimper et al. [19]; and SICStus Pro$\log$ [22]. Definition 5 appears in for example Marriott \& Stuckey [16]; Schulte \& Stuckey [21]; Harvey \& Schimpf [10]; and Zhang \& Yap [26]. Apt 1] gives both Definitions 4 and 5 calling the former interval consistency and the latter bounds consistency.

Let us now examine the differences of the definitions. Consider two notions of consistency $\Omega$ and $\Pi$. We say that $\Omega$ consistency is at least as strong as $\Pi$ consistency iff, given any constraint $c$ and domain $D$, if $D$ is $\Omega$ consistent for $c$ then $D$ is $\Pi$ consistent for $c$. We say that $\Omega$ consistency is stronger than $\Pi$ consistency iff $\Omega$ consistency is at least as strong as $\Pi$ consistency, but $\Pi$ consistency is not at least as strong as $\Omega$ consistency. The following relationship between the three notions of bounds consistency is clear from the definition.

Proposition 6 Bounds $(\mathcal{D})$ consistency is stronger than bounds $(\mathcal{Z})$ consistency, which is stronger than bounds $(\mathcal{R})$ consistency.

Proof: For each of the notions of consistency, for each bound of each variable a support must be found, i.e. an assignment of values to each of the other variables. For each of these other variables, let $S_{\mathcal{D}}, S_{\mathcal{Z}}$, and $S_{\mathcal{R}}$ be the set of allowed values to choose from for bounds $(\mathcal{D})$, bounds $(\mathcal{Z})$ and bounds $(\mathcal{R})$ consistency respectively.

From the definition, $S_{\mathcal{D}} \subseteq S_{\mathcal{Z}}$, thus having a support for bounds $(\mathcal{D})$ consistency implies having a support for bounds $(\mathcal{Z})$ consistency. The reverse is not always true: see Examplel7 Hence, bounds $(\mathcal{D})$ consistency is stronger than bounds $(\mathcal{Z})$ consistency. Similarly, $S_{\mathcal{Z}} \subseteq S_{\mathcal{R}}$, so bounds $(\mathcal{Z})$ consistency is stronger than bounds $(\mathcal{R})$ consistency (see Example 7 again for a case that is $\operatorname{bounds}(\mathcal{R})$ consistent but not bounds $(\mathcal{Z})$ consistent).

Example 7 Consider the constraint $c_{l i n}$ from Example 2 The domain $D_{2}$ defined by $D_{2}\left(x_{1}\right)=\{2,3,4,6,7\}, D_{2}\left(x_{2}\right)=[0 . .2]$, and $D_{2}\left(x_{3}\right)=[0 . .1]$ is bounds $(\mathcal{R})$ consistent (but not bounds $(\mathcal{D})$ consistent nor bounds $(\mathcal{Z})$ consistent) w.r.t. $c_{l i n}$, supported by the real solutions $\left\{x_{1} \mapsto 2, x_{2} \mapsto 2 / 3, x_{3} \mapsto 0\right\},\left\{x_{1} \mapsto\right.$ $\left.7, x_{2} \mapsto 2, x_{3} \mapsto 1 / 5\right\},\left\{x_{1} \mapsto 5, x_{2} \mapsto 0, x_{3} \mapsto 1\right\}$.

The domain $D_{3}$ defined by $D_{3}\left(x_{1}\right)=\{3,4,6\}, D_{3}\left(x_{2}\right)=[0 . .2]$, and $D_{3}\left(x_{3}\right)=$ $[0 . .1]$ is bounds $(\mathcal{Z})$ and bounds $(\mathcal{R})$ consistent (but not bounds $(\mathcal{D})$ consistent) w.r.t. $c_{l i n}$.

The domain $D_{4}$ defined by $D_{4}\left(x_{1}\right)=\{3,4,6\}, D_{4}\left(x_{2}\right)=[1 . .2]$, and $D_{4}\left(x_{3}\right)=$ $\{0\}$ is bounds $(\mathcal{D})$, bounds $(\mathcal{Z})$ and bounds $(\mathcal{R})$ consistent w.r.t. $c_{\text {lin }}$.

The relationship between the bounds $(\mathcal{Z})$ and bounds $(\mathcal{D})$ consistency is straightforward to explain.

Proposition $8 D$ is bounds $(\mathcal{Z})$ consistent with c iff $\operatorname{range}(D)$ is bounds $(\mathcal{D})$ consistent with $c$. 
The second definition of bounds consistency in Dechter [7] page 435] works only with range domains. By Proposition 8 the definition coincides with both bounds $(\mathcal{Z})$ and bounds $(\mathcal{D})$ consistency. Similarly, Apt's [1] interval consistency is also equivalent to bounds $(\mathcal{D})$ consistency. But in general, finite domain constraint solvers do not always operate on range domains, but rather use a mix of propagators implementing different kinds of consistencies, both domain and bounds consistency.

Example 9 Consider the constraint $c_{l i n}$ and domain $D_{3}$ from Example[7 Now range $\left(D_{3}\right)$ is both bounds $(\mathcal{D})$ and bounds $(\mathcal{Z})$ consistent with $c_{\text {lin }}$. But, as noted in Example $7 D_{3}$ is only bounds $(\mathcal{Z})$ consistent but not bounds $(\mathcal{D})$ consistent with $c_{\text {lin }}$.

Both bounds $(\mathcal{R})$ and bounds $(\mathcal{Z})$ consistency depend only on the upper and lower bounds of the domains of the variables under consideration.

Proposition 10 For $\alpha=\mathcal{R}$ or $\alpha=\mathcal{Z}$ and constraint $c, D$ is bounds $(\alpha)$ consistent for $c$ iff range $(D)$ is bounds $(\alpha)$ consistent for $c$.

This is not the case for bounds $(\mathcal{D})$ consistency, which suggests that, strictly, it is not really a form of bounds consistency. Indeed, most existing implementations of bounds propagators make use of Proposition 10 to avoid reexecuting a bounds propagator unless the lower or upper bound of a variable involved in the propagator changes.

Example 11 Consider the constraint $c_{l i n}$ and domain $D_{3}$ from Example $\mathbf{7}$ again. Both $D_{3}$ and range $\left(D_{3}\right)$ are bounds $(\mathcal{Z})$ and bounds $(\mathcal{R})$ consistency with $c_{\text {lin }}$, but only range $\left(D_{3}\right)$ is bounds $(\mathcal{D})$ consistent with $c_{\text {lin }}$.

There are significant problems with the stronger bounds $(\mathcal{Z})$ (and bounds $(\mathcal{D})$ ) consistency. In particular, for linear equations it is NP-complete to check bounds $(\mathcal{Z})$ (and bounds $(\mathcal{D})$ ) consistency, while for bounds $(\mathcal{R})$ consistency it is only linear time (e.g. see Schulte \& Stuckey [21]).

Proposition 12 Checking bounds $(\mathcal{Z})$, bounds $(\mathcal{D})$, or domain consistency of a domain $D$ with a linear equality $a_{1} x_{1}+\cdots a_{n} x_{n}=a_{0}$ where $\left\{a_{0}, \ldots, a_{n}\right\}$ are integer constants and $\left\{x_{1}, \ldots, x_{n}\right\}$ are integer variables, is NP-complete.

Proof: It is clear that this problem belongs to NP. Next, we show that the SUBSET SUM NP-complete problem [6 is polynomial reducible to this problem.

In the subset sum problem, we are given a set of $n$ natural numbers $A=\left\{a_{1}, \ldots, a_{n}\right\}$ and a target natural number $a_{n+1}$, and we must determine whether or not there exists a subset $S \subseteq A$ where $\sum\left\{a_{i} \mid a_{i} \in S \wedge 1 \leq i \leq n\right\}=a_{n+1}$. For each integer $a_{i}$ where $1 \leq i \leq n+1$ we associate an integer variable $x_{i}$ with the domain $D$ defined by $D\left(x_{i}\right)=\{0,1\}$. We also introduce another integer 
variable $x_{n+2}$ where $D\left(x_{n+2}\right)=\{0,1\}$. Now, the linear equation $c$ defined by

$$
a_{1} x_{1}+\cdots+a_{n} x_{n}-a_{n+1} x_{n+1}-\left(\sum_{i=1}^{n} a_{i}\right) x_{n+2}=0
$$

has integer solutions:

$$
\begin{aligned}
& \left\{x_{1} \mapsto 0, \ldots, x_{n} \mapsto 0, x_{n+1} \mapsto 0, x_{n+2} \mapsto 0\right\} \\
& \left\{x_{1} \mapsto 1, \ldots, x_{n} \mapsto 1, x_{n+1} \mapsto 0, x_{n+2} \mapsto 1\right\}
\end{aligned}
$$

These two solutions exhibit that all domain elements for all variables have support, with the exception of $x_{n+1} \mapsto 1$. Thus, $D$ is domain consistent for $c$ iff there exists a valuation $\theta \in D$ where $\theta\left(x_{n+1}\right)=1$ that satisfies $c$. Clearly we cannot have $\theta\left(x_{n+1}\right)=1$ and $\theta\left(x_{n+2}\right)=$ 1 (no solution), so any such $\theta$ must have $\theta\left(x_{n+2}\right)=0$. Such a valuation, if it exists, defines a set $S=\left\{a_{i} \mid \theta\left(x_{i}\right)=1 \wedge 1 \leq i \leq n\right\}$ such that $\sum\left\{a_{i} \mid a_{i} \in S \wedge 1 \leq i \leq n\right\}=a_{n+1}$; conversely, the existence of such a set implies a corresponding valuation. Thus there exists a solution to the SUBSET SUM problem iff there exists a solution of $c$ with $\theta\left(x_{n+1}\right)=1$ and $\theta\left(x_{n+2}\right)=0$, iff $D$ is domain consistent with respect to $c$.

For domain $D$, checking bounds $(\mathcal{Z})$, bounds $(\mathcal{D})$, or domain consistency for $c$ are equivalent. Hence, the cases for checking bounds $(\mathcal{Z})$ and bounds $(\mathcal{D})$ consistency follow analogously.

There are constraints, however, for which $\operatorname{bounds}(\mathcal{R})$ consistency is less meaningful than other forms of consistency.

Example 13 Consider the global constraint alldifferent $\left(x_{1}, x_{2}, x_{3}\right) \equiv x_{1} \neq$ $x_{2} \wedge x_{1} \neq x_{3} \wedge x_{2} \neq x_{3}$ and the domain $D_{5}$ where $D_{5}\left(x_{1}\right)=D_{5}\left(x_{2}\right)=[1 . .2]$ and $D_{5}\left(x_{3}\right)=[2 \ldots 3]$. Then this domain is not bounds $(\mathcal{Z})$ consistent, since there is no integer solution with $x_{3}=2$. But the domain $D_{5}$ is bounds $(\mathcal{R})$ consistent with alldifferent $\left(x_{1}, x_{2}, x_{3}\right)$ since it has real solutions $\left\{x_{1} \mapsto 1, x_{2} \mapsto 2, x_{3} \mapsto\right.$ $3\},\left\{x_{1} \mapsto 2, x_{2} \mapsto 1, x_{3} \mapsto 3\right\},\left\{x_{1} \mapsto 1, x_{2} \mapsto 1.5, x_{3} \mapsto 2\right\}$.

A problem with bounds $(\mathcal{R})$ consistency is that it may not be clear how to interpret an integer constraint in the reals.

Example 14 Consider the constraint $x_{1}=x_{2} \bmod x_{3}$. While this constraint is well-defined for integer values of the variables, in order to check bounds $(\mathcal{R})$ consistency we need to clarify what it means to be a real solution of $x_{1}=x_{2}$ $\bmod x_{3}$.

Example 15 Consider the reified constraint $b \Leftrightarrow x_{1}+x_{2} \leq x_{3}$, where $b=1$ corresponds to $x_{1}+x_{2} \leq x_{3}$ being true, and $b=0$ corresponds to it being false. However, it is not clear what natural interpretation can be given to, say, $b=0.5$. 


\section{Conditions for Equivalence}

Why has the confusion between the various definitions of bounds consistency not been noted before? In fact, for many constraints, the definitions are equivalent.

Following the work of Zhang \& Yap [26] we define $n$-ary monotonic constraints as a generalization of linear inequalities $\sum_{i=1}^{n} a_{i} x_{i} \leq a_{0}$. Let $\theta \in \mathcal{R} D$ denote that $\theta(v) \in \mathcal{R}$ and $\inf _{D} v \leq \theta(v) \leq \sup _{D} v$ for all $v \in \operatorname{vars}(\theta)$.

Definition 16 An $n$-ary constraint $c$ is monotonic with respect to variable $x_{i} \in \operatorname{vars}(c)$ iff there exists a total ordering $\prec_{i}$ on $D\left(x_{i}\right)$ such that if $\theta \in \mathcal{R} D$ is a real solution of $c$, then so is any $\theta^{\prime} \in_{\mathcal{R}} D$ where $\theta^{\prime}\left(x_{j}\right)=\theta\left(x_{j}\right)$ for $j \neq i$ and $\theta^{\prime}\left(x_{i}\right) \preceq_{i} \theta\left(x_{i}\right)$.

An $n$-ary constraint $c$ is monotonic iff $c$ is monotonic with respect to all variables in $\operatorname{vars}(c)$.

The above definition of monotonic constraints is equivalent to but simpler than that of Zhang \& Yap [26]; see Choi et al. [5] for justification and explanation. In the following, we assume that $\prec_{i}$ is $<$ or $>$ for all $i$, in order to restrict ourselves to orders which are sensible with respect to bounds consistency.

Examples of such monotonic constraints are:

- all linear inequalities

- $x_{1} \times x_{2} \leq x_{3}$ with non-negative domains, e.g. $D\left(x_{i}\right)=[1 . .1000]$ for $1 \leq i \leq 3$

For this class of constraints, bounds $(\mathcal{R})$, bounds $(\mathcal{Z})$ and bounds $(\mathcal{D})$ consistency are equivalent to domain consistency.

Proposition 17 Let $c$ be an n-ary monotonic constraint. Then bounds(R), bounds $(\mathcal{Z})$, bounds $(\mathcal{D})$ and domain consistency for $c$ are all equivalent.

Proof: Since domain consistency $\Rightarrow \operatorname{bounds}(\mathcal{D})$ consistency $\Rightarrow \operatorname{bounds}(\mathcal{Z})$ consistency $\Rightarrow$ bounds $(\mathcal{R})$ consistency, it is sufficient to show here that bounds $(\mathcal{R})$ consistency implies domain consistency for this constraint; thus we assume $D$ is $\operatorname{bounds}(\mathcal{R})$ consistent and prove it is also domain consistent.

Consider the greatest value $d_{i} \in D\left(x_{i}\right)$ for some $x_{i} \in \operatorname{vars}(c)$ (i.e. $d_{i}$ must be either $\inf _{D} x_{i}$ or $\sup _{D} x_{i}$ depending on the ordering $\prec_{i}$ that makes $c$ monotonic w.r.t. $\left.x_{i}\right)$. Since $D$ is bounds $(\mathcal{R})$ consistent, there exists $\theta \in \mathcal{R} D$ with $\theta\left(x_{i}\right)=d_{i}$ such that $\theta$ is a real solution of $c$.

Let $\theta^{\prime}\left(x_{i}\right)=\theta\left(x_{i}\right)$ and $\theta^{\prime}\left(x_{j}\right)=d_{j}$ for all $j \neq i$ where $d_{j}$ is the smallest element of $D\left(x_{j}\right)$ with respect to $\prec_{j}$. Since $c$ is monotonic, $\theta^{\prime}$ is a solution of $c$, and since the smallest elements of the domains are necessarily integers, $\theta^{\prime}$ must be an integer solution of $c$. 
Now consider $\theta^{\prime \prime}$, where $\theta^{\prime \prime}\left(x_{j}\right)=\theta^{\prime}\left(x_{j}\right)$ for all $j \neq i$ and $\theta^{\prime \prime}\left(x_{i}\right)=d$ for some $d \in D\left(x_{i}\right)$. Since $c$ is monotonic and $d \preceq_{i} \theta^{\prime}\left(x_{i}\right)$ (since $\theta^{\prime}\left(x_{i}\right)$ is the largest element of $\left.D\left(x_{i}\right)\right), \theta^{\prime \prime}$ is also an integer solution of $c$, with $\theta^{\prime \prime}\left(x_{i}\right)=d$.

Since the choice of $x_{i}$ and $d$ was arbitrary, we have shown that we can construct an integer solution supporting any value in the domain of any variable; thus $D$ is domain consistent.

Although disequality constraints are not monotonic, they are equivalent for all the forms of bounds consistency because they prune so weakly.

Proposition 18 Let $c \equiv \sum_{i=1}^{n} a_{i} x_{i} \neq a_{0}$. Then bounds $(\mathcal{R})$, bounds $(\mathcal{Z})$ and bounds $(\mathcal{D})$ consistency for $c$ are equivalent.

Proof: (Sketch) Essentially if a value $d$ of a variable $x_{i}$ where $1 \leq$ $i \leq n$ is supported by $c$, it is supported by an integer value in $D\left(x_{i}\right)$. Since either the domain of a variable is a singleton, in which case the bounds consistency must use the unique integer, or the domain is not a singleton, and one of the two integral endpoints will suffice.

All forms of bounds consistency are also equivalent for binary functional constraints, such as $a_{1} x_{1}+a_{2} x_{2}=a_{0}, x_{1}=a x_{2}{ }^{2} \wedge x_{2} \geq 0$, or $x_{1}=1+x_{2}+$ $x_{2}{ }^{2}+x_{2}{ }^{3} \wedge x_{2} \geq 0$.

Proposition 19 Let $c$ be a constraint with vars $(c)=\left\{x_{1}, x_{2}\right\}$, where $c \equiv x_{1}=$ $g\left(x_{2}\right)$ and $g$ is a bijective and monotonic function. Then bounds $(\mathcal{R})$, bounds $(\mathcal{Z})$ and bounds $(\mathcal{D})$ consistency for $c$ are equivalent.

Proof: Assume $D$ is bounds $(\mathcal{R})$ consistent w.r.t. $c$. Let us examine the endpoints of the ranges of each variable. We will examine $d_{1}=$ $\inf _{D} x_{1}$, and assume $g$ is monotonically increasing. The other cases are similar.

Now $\left\{x_{1} \mapsto d_{1}, x_{2} \mapsto g\left(d_{1}\right)\right\}$ is the unique real solution of $c$ supporting $d_{1}$. Hence $\inf _{D} x_{2} \leq g\left(d_{1}\right) \leq \sup _{D} x_{2}$. Suppose that $g\left(d_{1}\right)>$ $\inf _{D} x_{2}=d_{2}$. Then $d_{1}=g^{-1}\left(g\left(d_{1}\right)\right)>g^{-1}\left(d_{2}\right)$ since $g^{-1}$ is also monotonically increasing. Hence $d_{2}$ cannot be supported by any value in the domain of $x_{1}$. Contradiction. Thus $d_{2}=g\left(d_{1}\right)=$ $\inf _{D} x_{2}$. Both $d_{1} \in D\left(x_{1}\right)$ and $d_{2} \in D\left(x_{2}\right)$ are integers since they are bounds. Hence $\left\{x_{1} \mapsto d_{1}, x_{2} \mapsto g\left(d_{1}\right)\right\}$ is an integer solution supporting $d_{1}$ for $x_{1}$. Since the supporting solution only involves the end points in this case bounds $(\mathcal{Z})$ and bounds $(\mathcal{D})$ are equivalent.

The proofs of Propositions 17 18, and 19 are related to but different from the proofs of endpoint-relevance for these constraints in Schulte \& Stuckey [21].

For linear equations with at most one non-unit coefficient, we can show that bounds $(\mathcal{R})$ and bounds $(\mathcal{Z})$ consistency are equivalent. 
Proposition 20 Let $c \equiv \sum_{i=1}^{n} a_{i} x_{i}=a_{0}$, where $\left|a_{i}\right|=1,2 \leq i \leq n, a_{0}$ and $a_{1}$ integral. Then bounds $(\mathcal{R})$ and bounds $(\mathcal{Z})$ consistency for $c$ are equivalent.

Proof: $\operatorname{Bounds}(\mathcal{Z})$ consistency implies bounds $(\mathcal{R})$ consistency, so it is sufficient to show here that bounds $(\mathcal{R})$ consistency implies bounds $(\mathcal{Z})$ consistency for this constraint; thus we assume $D$ is bounds $(\mathcal{R})$ consistent and prove it is also bounds $(\mathcal{Z})$ consistent.

First consider a bound of $x_{1}$, say $d_{1}=\inf _{D} x_{1}$ (the other bound follows analogously). Since $D$ is bounds $(\mathcal{R})$ consistent, there exist real $d_{i}, \inf _{D} x_{i} \leq d_{i} \leq \sup _{D} x_{i}, 2 \leq i \leq n$ such that $\left\{x_{i} \mapsto d_{i} \mid 1 \leq\right.$ $i \leq n\}$ is a real solution of $c$. Note that $\sum_{i=2}^{n} a_{i} d_{i}$ is integral, and since $\left|a_{i}\right|=1(2 \leq i \leq n)$, it is straightforward to construct integral $d_{i}^{\prime}$ with the same sum that respect the variables' bounds. ${ }^{1}$ These $d_{i}^{\prime}$ exhibit that the bound $d_{1}$ is bounds $(\mathcal{Z})$ consistent.

Note that the fact that $x_{1}$ is $\operatorname{bounds}(\mathcal{R})$ consistent also implies

$$
\inf _{D} a_{1} x_{1} \geq a_{0}-\sum_{i=2}^{n} \sup _{D} a_{i} x_{i}
$$

Now consider a bound of any of the other variables. For simplicity we assume $a_{2}=1$ and consider the upper bound of $x_{2}$; the other cases follow analogously. Let $e_{2}=\sup _{D} x_{2}$. Since $D$ is bounds $(\mathcal{R})$ consistent, there exist real $e_{i}, \inf _{D} x_{i} \leq e_{i} \leq \sup _{D} x_{i}, i \in\{1,3 \ldots n\}$ such that $\left\{x_{i} \mapsto e_{i} \mid 1 \leq i \leq n\right\}$ is a real solution of $c$. In particular, this means that

$$
\sup _{D} x_{2} \leq a_{0}-\inf _{D} a_{1} x_{1}-\sum_{i=3}^{n} \inf _{D} a_{i} x_{i}
$$

but (11) implies

$$
\sup _{D} x_{2} \geq a_{0}-\inf _{D} a_{1} x_{1}-\sum_{i=3}^{n} \sup _{D} a_{i} x_{i}
$$

This means $\sup _{D} x_{2}=a_{0}-\inf _{D} a_{1} x_{1}-k$ where $k$ is an integer and $\sum_{i=3}^{n} \inf _{D} a_{i} x_{i} \leq k \leq \sum_{i=3}^{n} \sup _{D} a_{i} x_{i}$. Clearly we can find integer values $f_{i}$ to assign to $x_{i}(3 \leq i \leq n)$ to make their sum equal to $k$ while satisfying the variable bounds, since the corresponding $a_{i}$ are \pm 1 . If we let $f_{2}=\sup _{D} x_{2}$ and let $f_{1}$ be such that $a_{1} f_{1}=\inf _{D} a_{1} x_{1}$, then $\left\{x_{i} \mapsto f_{i}, 1 \leq i \leq n\right\}$ is an integer solution of $c$ that exhibits that $\sup _{D} x_{2}$ is bounds $(\mathcal{Z})$ consistent.

Even for linear equations with all unit coefficients, bounds $(\mathcal{D})$ consistency is different from bounds $(\mathcal{Z})$ and bounds $(\mathcal{R})$ consistency.

\footnotetext{
${ }^{1}$ For example, compute the average fractional part of the non-integral $d_{i} \mathrm{~s}$, and then round that fraction of them up to the next integer; the rest are rounded down. This clearly respects the variable bounds, because they are integral and we never change a value beyond an integer.
} 
Example 21 [Counter example] Consider the linear equation $c \equiv x_{1}+x_{2}+x_{3}=$ 5 and the domain $D_{8}$ where $D_{8}\left(x_{1}\right)=[0 . .3], D_{8}\left(x_{2}\right)=D_{8}\left(x_{3}\right)=\{0,3,4,5\}$. $D_{8}$ is bounds $(\mathcal{R})$ and bounds $(\mathcal{Z})$ consistent as given by the following solutions of $c:\left\{x_{1} \mapsto 0, x_{2} \mapsto 0, x_{3} \mapsto 5\right\},\left\{x_{1} \mapsto 0, x_{2} \mapsto 5, x_{3} \mapsto 0\right\}$, and $\left\{x_{1} \mapsto 3, x_{2} \mapsto\right.$ $\left.1, x_{3} \mapsto 1\right\}$. $D_{8}$ is, however, not bounds $(\mathcal{D})$ consistent, since there is clearly no integer solution with $x_{1} \mapsto 3$ (i.e. neither 1 nor 2 are in the domains of $x_{2}$ and $\left.x_{3}\right)$.

In summary for many of the constraints commonly used the notions of bounds consistency are equivalent, but clearly not for all.

\section{Related Work}

In this paper we restricted ourselves to integer constraint solving. Definitions of bounds consistency for real constraints are also numerous, but their similarities and differences have been noted and explained by e.g. Benhamou et al. 2]. Indeed, we can always interpret integers as reals and apply bounds consistency for real constraints plus appropriate rounding, e.g. CLP(BNR) 3]. However, as we have pointed out in Section 3 there exist integer constraints for which propagation is less meaningful when interpreted as reals.

Lhomme 13 defines arc B-consistency which formalizes bounds propagation techniques for numeric CSPs. Unlike our definition of CSPs, constraints in numeric CSPs cannot be given extensionally and must be defined by numeric relations, which can be interpreted in either the real or the finite integer domain. Numeric CSPs also restrict the domain of variables to be a single interval.

Walsh 24] introduces several new forms of bounds consistency which extends the notion of $(i, j)$-consistency and relational consistency. He gives a theoretical analysis comparing the propagation strength of these new forms of bounds consistency.

Maher [15] introduces the notion of propagation completeness together with a general framework to unify a wide range of consistency. These include hull consistency of real constraints and bounds $(\mathcal{Z})$ consistency of integer constraints. Propagation completeness aims to capture the timeliness property of propagation.

The application of bounds consistency is not limited to integer and real constraints. Bounds consistency has been formalized for solving set constraints 9], and more recently, multiset constraints [25].

\section{Conclusion}

The contributions of this paper are two-fold. First, we point out that the three commonly used definitions of bounds consistency are incompatible. Second, we clarify their differences and show that for several types of constraints, bounds $(\mathcal{R})$, bounds $(\mathcal{Z})$ and bounds $(\mathcal{D})$ consistency are equivalent. This explains partly why the discrepancies among the definitions were not noticed 
earlier. Our precise definitions can serve as the basis for verifying all implementations of bounds propagators.

\section{References}

[1] K. Apt. Principles of Constraint Programming. Cambridge University Press, 2003.

[2] F. Benhamou, D. McAllester, and P. Van Hentenryck. CLP(Intervals) revisited. In ILPS 1994, pages 124-138, 1994.

[3] F. Benhamou and W. J. Older. Applying interval arithmetic to real, integer, and boolean constraints. JLP, 32(1):1-24, 1997.

[4] A. M. Cheadle, W. Harvey, A. J. Sadler, J. Schimpf, K. Shen, and M. G. Wallace. ECL ${ }^{i} \mathrm{PS}^{e}$ : An introduction. Technical Report IC-Parc-03-1, ICParc, Imperial College London, 2003.

[5] C. W. Choi, W. Harvey, J. H. M. Lee, and P. J. Stuckey. A note on the definition of constraint monotonicity. Available from http://www.cse.cuhk.edu.hk/ cwchoi/monotonicity.pdf 2004.

[6] T. H. Cormen, C. E. Leiserson, and R. L. Rivest. Introduction to Algorithms. The MIT Press, 1990.

[7] R. Dechter. Constraint Processing. Morgan Kaufmann, 2003.

[8] A. Frisch, B. Hnich, Z. Kiziltan, I. Miguel, and T. Walsh. Global constraints for lexicographic orderings. In CP2002, pages 93-108. Springer-Verlag, 2002.

[9] C. Gervet. Interval propagation to reason about sets: Definition and implementation of a practical language. Constraints, 1(3):191-244, 1997.

[10] W. Harvey and J. Schimpf. Bounds consistency techniques for long linear constraints. In Proceedings of TRICS: Techniques foR Implementing Constraint programming Systems, pages 39-46, 2002.

[11] ILOG Solver. ILOG Solver 5.2: User's Manual, 2001.

[12] A. Lallouet, A. Legtchenko, T. Dao, and A. Ed-Dbali. Intermediate (learned) consistencies. Research Report RR-LIFO-2003-04, Laboratoire d'Informatique Fondamentale d'Orléans, 2003.

[13] O. Lhomme. Consistency techniques for numeric CSPs. In IJCAI-93, pages 232-238, 1993.

[14] A. K. Mackworth. Consistency in networks of relations. AI Journal, 8(1):99-118, 1977. 
[15] M. Maher. Propagation completeness of reactive constraints. In ICLP 2002, pages 148-162, 2002.

[16] K. Marriott and P. J. Stuckey. Programming with Constraints: an Introduction. The MIT Press, 1998.

[17] R. Mohr and G. Masini. Good old discrete relaxation. In ECAI-88, pages 651-656, 1998.

[18] J.-F. Puget. A fast algorithm for the bound consistency of alldiff constraints. In AAAI-98, pages 359-366, 1998.

[19] C.-G. Quimper, P. van Beek, A. López-Ortiz, A. Golynski, and S. B. Sadjad. An efficient bounds consistency algorithm for the global cardinality constraint. In CP 2003, pages 600-614, 2003.

[20] J.-C. Régin and M. Rueher. A global constraint combining a sum constraint and difference constraints. In CP 2000, pages 384-395, 2000.

[21] C. Schulte and P. J. Stuckey. When do bounds and domain propagation lead to the same search space. In PPDP 2001, pages 115-126, 2001.

[22] SICStus Prolog. SICStus Prolog User's Manual, Release 3.10.1, 2003.

[23] P. Van Hentenryck, V. Saraswat, and Y. Deville. Design, implementation and evaluation of the constraint language cc(FD). JLP, 37(1-3):139-164, 1998.

[24] T. Walsh. Relational consistencies. Research Report APES-28-2001, APES Research Group, 2001.

[25] T. Walsh. Consistency and propagation with multiset constraints: A formal viewpoint. In $C P$ 2003, 2003. 724-738.

[26] Y. Zhang and R. H. C. Yap. Arc consistency on $n$-ary monotonic and linear constraints. In $C P$ 2000, pages 470-483, 2000. 